\title{
The Role of Congruency for Distractor-Response Binding: A Caveat
}

\author{
Roland Pfister' Christian Frings'2, and Birte Moeller ${ }^{2}$, \\ 'Julius Maximilians University of Würzburg, Department of Psychology III, Röntgenring 11, 97070 Würzburg, Germany \\ ${ }^{2}$ University of Trier, Department of Psychology, Universitätsring 15, 54296 Trier, Germany
}

\section{KEYWORDS}

action control

distractor-response binding

congruency sequences

sequence analysis
ABSTRACT

Responding in the presence of stimuli leads to an integration of stimulus features and response features into event files, which can later be retrieved to assist action control. This integration mechanism is not limited to target stimuli, but can also include distractors (distractor-response binding). A recurring research question is which factors determine whether or not distractors are integrated. One suggested candidate factor is target-distractor congruency: Distractor-response binding effects were reported to be stronger for congruent than for incongruent target-distractor pairs. Here, we discuss a general problem with including the factor of congruency in typical analyses used to study distractor-based binding effects. Integrating this factor leads to a confound that may explain any differences between distractor-response binding effects of congruent and incongruent distractors with a simple congruency effect. Simulation data confirmed this argument. We propose to interpret previous data cautiously and discuss potential avenues to circumvent this problem in the future.

\section{INTRODUCTION}

Successfully performing an action leaves a lasting trace in the cognitive system: Features that were used to represent, plan, and initiate the action are automatically integrated with features of the current situation, and the structures that include these bindings of stimulus- and response-features are often labeled event files (Frings et al., in press; Hommel, 1998, 2004).

An elegant way to study binding and retrieval of event files is the distractor-response binding paradigm (Frings, Rothermund, \& Wentura, 2007). In this paradigm, participants typically perform a choice reaction time task in which targets (e.g., letter stimuli) call for one of two responses (e.g., left vs. right keypress) while targets are accompanied by distractor stimuli (e.g., flanker stimuli to the left and right of the target). Crucially, these experiments aim at studying sequential dependencies for two successive responses, either by explicitly implementing a prime-probe sequence or by analyzing sequential effects for a continuous series of responses (Moeller, Frings, \& Pfister, 2016). Irrespective of the precise design used, we refer to the currently analyzed trial as probe and its immediately preceding trial as prime trial in the following argument.

Sequential analyses of such designs allow for measuring distractorresponse binding by modeling performance (response times and error

Corresponding author: Roland Pfister, Julius Maximilians University of Würzburg, Department of Psychology III, Röntgenring 11, 97070 Würzburg, Germany.

e-mail: roland.pfister@psychologie.uni-wuerzburg.de 
percentages) as a joint function of the response sequence (repetition vs. alternation) and the distractor sequence (repetition vs. alternation) from prime to probe. Distractor repetitions typically improve performance for response repetitions whereas distractor repetitions impair performance of response alternations (relative to distractor alternations). This pattern of results is taken as evidence that features of the distractor and the response become integrated in an event file during the prime, which is retrieved in the probe if one of the features is reactivated, for example, by repeating the distractor stimulus from prime to probe (Hommel, 1998; Rothermund, Wentura, \& De Houwer, 2005). In technical terms, distractor-response binding thus becomes evident as an interaction of the response sequence factor and the distractor sequence factor, and the presence and strength of this interaction can be used to study relevant preconditions and moderators of event-file binding and retrieval.

In addition to distractor and response features, event files further incorporate additional features relating to the target stimulus and possible effects of the response (Bogon, Thomaschke, \& Dreisbach, 2017; Colzato, van Wouwe, \& Hommel, 2007; Dutzi \& Hommel, 2009; Giesen \& Rothermund, 2011; Hommel, 2005; Huffman, Hilchey, \& Pratt, in press; Janczyk, Heinemann, \& Pfister, 2012; Moeller, Pfister, Kunde, \& Frings, 2016; Schwarz, Burger, Dignath, Kunde, \& Pfister, 2018). Crucially, however, bindings are often assumed to be binary, that is, event files are conceptualized as a set of bindings between feature pairs which are independent of bindings between other features in the event file. Therefore, it is reasonable to consider pairwise bindings individually, and we focus on distractor-response bindings in the following

The literature on distractor-response binding describes a wide range of preconditions and moderators of binding and retrieval, such as perceptual grouping (Frings \& Rothermund, 2011; Moeller, Rothermund, \& Frings, 2012), attentional allocation (Hommel, Memelink, Zmigrod, \& Colzato, 2014; Moeller \& Frings, 2014a), or temporal decay (Frings, 2011; Moeller, Pfister et al., 2016). One particular moderator for binding and retrieval which has been proposed in previous work is the congruency of distractor and target stimulus (Frings et al., 2007; Moeller \& Frings, 2014b). This situation is especially relevant when distractors and targets come from the same item pool so that the relevant mapping rule can be applied not only to the target but also to the distractor stimuli (e.g., Davelaar, 2013; Frings, 2011; Wiswede, Rothermund, \& Frings, 2013). Here, the interesting question is whether the integration of distractor and responses during the prime is impaired if distractorand target-associated responses are in conflict, that is, for incongruent prime trials as compared to congruent prime trials.

\section{DOES TARGET-DISTRACTOR CONGRUEN- CY MODERATE DISTRACTOR-RESPONSE BINDING?}

A tempting approach to answer this question is to compute distractor response binding separately for congruent and incongruent prime trials so that the three-way interaction of prime congruency, response sequence, and distractor sequence informs about differences in distractor-response binding depending on congruency during the integration of distractor and response. At first sight, this approach yielded clearcut results by showing strong and robust distractor-response binding for congruent prime trials but reduced and numerically even reversed binding for incongruent prime trials (Moeller \& Frings, 2014b).

On closer inspection, however, this methodology confounds the assumed moderating role of prime congruency (i.e., the three-way interaction of prime congruency, response sequence, and distractor sequence) with target-distractor congruency in the probe trial. To illustrate this point, we will assume the same design as used by Moeller and Frings (2014b), in which target letters were flanked by triplets of arrowheads which pointed either to the left or to the right (e.g., " $<<<$ F $<<")$. Figure 1 breaks down the individual trial sequences according to the differential contribution of congruent and incongruent probe trials. Following this argument, the three-way interaction of prime congruency, response sequence, and distractor sequence actually is (at least partially) a disguised measure of probe congruency.

The fundamental problem in comparing distractor-response binding effects for congruent and incongruent primes (or probes, for that matter) is that in typical experimental setups, each stimulus is compatible to a single response. Consequently, alternating the distractor category entails alternation of the associated response, meaning that distractor sequence, response sequence, and congruency in prime and probe are not independent. For example, if the distractor-associated and actually performed response are congruent during the prime, probe distractor and response are always congruent if both response and distractor are repeated or both are alternated, but they are incongruent if either distractor or response are alternated while the other repeats. Of course, the same logic holds for distractor/ response-incongruent primes. ${ }^{2}$ For the sketched experimental design, it is therefore impossible to differentiate any effect of congruency on distractor- and response integration in the prime, from a mere effect of distractor-response congruency during the probe. Both predict a less pronounced data pattern that is typical for distractor-response binding for incongruent than for congruent primes. ${ }^{3}$

To demonstrate the impact of this confound, we simulated data for 20 fictitious studies (the simulation code and the results that were used for all following analyses are available on the Open Science Framework, osf.io/d3gk2/). ${ }^{4}$ These studies were set to have 42 participants each, with 200 trials for each participant. Targets and distractors were sampled randomly with replacement for a vector of 200 consecutive trials. These trials were then coded as congruent if target and distractors were associated to the same response whereas trials were coded as incongruent if target and distractors were associated to opposite responses.

To generate the data, we used a simple model that only drew on the individual mean response time, $\overline{R T}$, a hypothetical congruency effect in the probe trial, and a random error term. $\overline{R T}$ was sampled from a normal distribution with a mean of $500 \mathrm{~ms}$ and a $S D$ of 100 ms: $\overline{R T} \sim \mathrm{N}(500,100)$. It was constant for each participant and RTs for individual trials of this participant were sampled from a normal distribution with this mean and a standard deviation of $100 \mathrm{~ms}$. We 


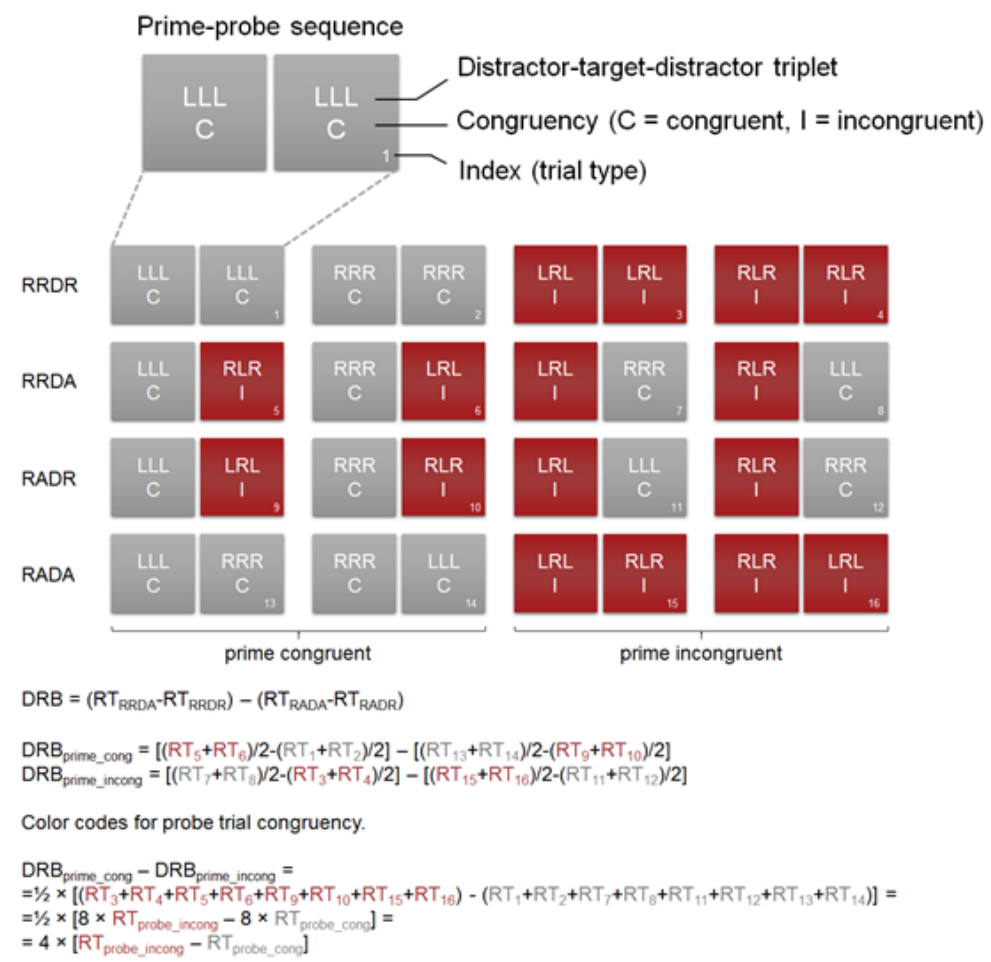

FIGURE 1.

Sketch of possible prime-probe sequences of a distractor-response binding paradigm in which a central target letter is flanked by two distractors to the left and right. Target and distractors can be associated either with a left response $(L)$ or a right response $(R)$; the displayed letter triplets thus represent the associated response of both target (center) and distractors (flankers) and the figure assumes a compact experimental design with only one distractor for left and right responses, respectively (following the design of Moeller \& Frings, 2014b). Each prime-probe sequence is classified as one of four conditions: response repetition and distractor repetition (RRDR), response repetition and distractor alternation (RRDA), response alternation and distractor repetition (RADR), or response alternation and distractor alternation (RADA). Mean RTs and error percentages in these conditions can be used to calculate distractorresponse binding effects (DRB) as detailed in the formulas for mean RT (the last two formulas require an equal number of trials per trial type to yield exact results). Color coding of the individual trial types codes the congruency of the corresponding probe trials with incongruent probe trials being printed in dark red and congruent probe trials being printed in light grey.

then determined randomly for each trial whether it was congruent or incongruent $\left(p_{\text {congruent }}=p_{\text {incongruent }}=0.5\right)$, and computed the current response time $R T_{\mathrm{i}}$ (with $1 \leq \mathrm{i} \leq 200$ ) as

$$
R T_{i}= \begin{cases}N(\overline{R T}, 100) & \text { for congruent probe trials } \\ N(\overline{R T}, 100)+N(20,200) & \text { for incongruent probe trials }\end{cases}
$$

Importantly, this model did not include any effect of distractorresponse binding or a modulation thereof by prime trial congruency. To verify that the simulation worked as intended, we analyzed the first simulated study with a repeated-measures analysis of variance (ANOVA) with the factors of probe congruency (congruent vs. incongruent), response sequence (repetition vs. alternation), and distractor sequence (repetition vs. alternation). The results of this analysis indicated that the model worked as intended by producing a pronounced effect of probe congruency and no further main effects or interactions (Table 1, left results). When coding for prime congruency instead of probe congruency, however, the modelled probe congruency effect reappeared as the three-way interaction of prime congruency, response sequence, and distractor sequence, spuriously suggesting a modulation of distractor-response binding by prime congruency (Table 1, right results). Accordingly, each of the 20 simulated studies yielded a large difference between the distractor-response binding effects computed for congruent and incongruent prime trials (see Figure 2 for the first 10 studies).

The above argument and demonstration suggest that typical binding paradigms are inappropriate to compare binding effects for congruent and incongruent distractor stimuli. Yet, the question whether an existing (congruent or incongruent) association between stimulus and response influences stimulus-response binding, and thus mechanisms of action control, remains an important one. For example, if one is interested in the mutual influence of binding and learning processes (e.g., Colzato, Raffone, \& Hommel, 2006; Herwig \& Waszak, 2012; Moeller \& Frings, 2017a), one might want to compare binding at the beginning and after a process of stimulus-response association.

One way to circumvent the described problem and analyze at least part of the influence congruency has on binding in action control, is to focus on exclusively incongruent pairings. Each stimulus is incongruent with multiple responses in the typical binding paradigms, allowing repetition of incongruency independent of distractor- and response sequence (Mordkoff, 2012). Results for incongruent conditions can then be com- 
TABLE 1.

Validation of the Argument via Simulation Results

\begin{tabular}{lrr}
\hline \multicolumn{3}{c}{ Model check: Probe Congruency } \\
\hline Source & $F(\mathbf{1 , 1 4})$ & $p$ \\
Probe Congruency [ProbeC] & 48.11 & .001 \\
Response Sequence [RS] & 1.07 & .307 \\
Distractor Sequence [DS] & 0.11 & .740 \\
ProbeC $\times$ RS & 0.28 & .598 \\
ProbeC $\times$ DS & 0.19 & .666 \\
RS $\times$ DS & 0.41 & .524 \\
ProbeC $\times$ RS $\times$ DS & 0.13 & .718 \\
\hline
\end{tabular}

\begin{tabular}{lrr}
\hline \multicolumn{3}{c}{ Prime congruency } \\
\hline Source & $F(\mathbf{1}, \mathbf{4 1})$ & $p$ \\
Prime Congruency [PrimeC] & 0.13 & .718 \\
Response Sequence [RS] & 1.07 & .307 \\
Distractor Sequence [DS] & 0.11 & .740 \\
PrimeC $\times$ RS & 0.19 & .666 \\
PrimeC $\times$ DS & 0.28 & .598 \\
RS $\times$ DS & 0.41 & .524 \\
PrimeC $\times$ RS $\times$ DS & 48.11 & .001 \\
\hline
\end{tabular}

Note. The simulation only assumed a congruency effect in the probe trial but no modulation of probe response times by the preceding prime trial (i.e., no distractorresponse binding). An analysis of variance (ANOVA) with the factors of congruency in the probe trial, response sequence, and distractor sequence yielded a reliable congruency effect and no other significant results. When using prime congruency instead of probe congruency, however, the congruency effect of the probe-trial reappears as the three-way interaction of prime congruency, response sequence, and distractor sequence.

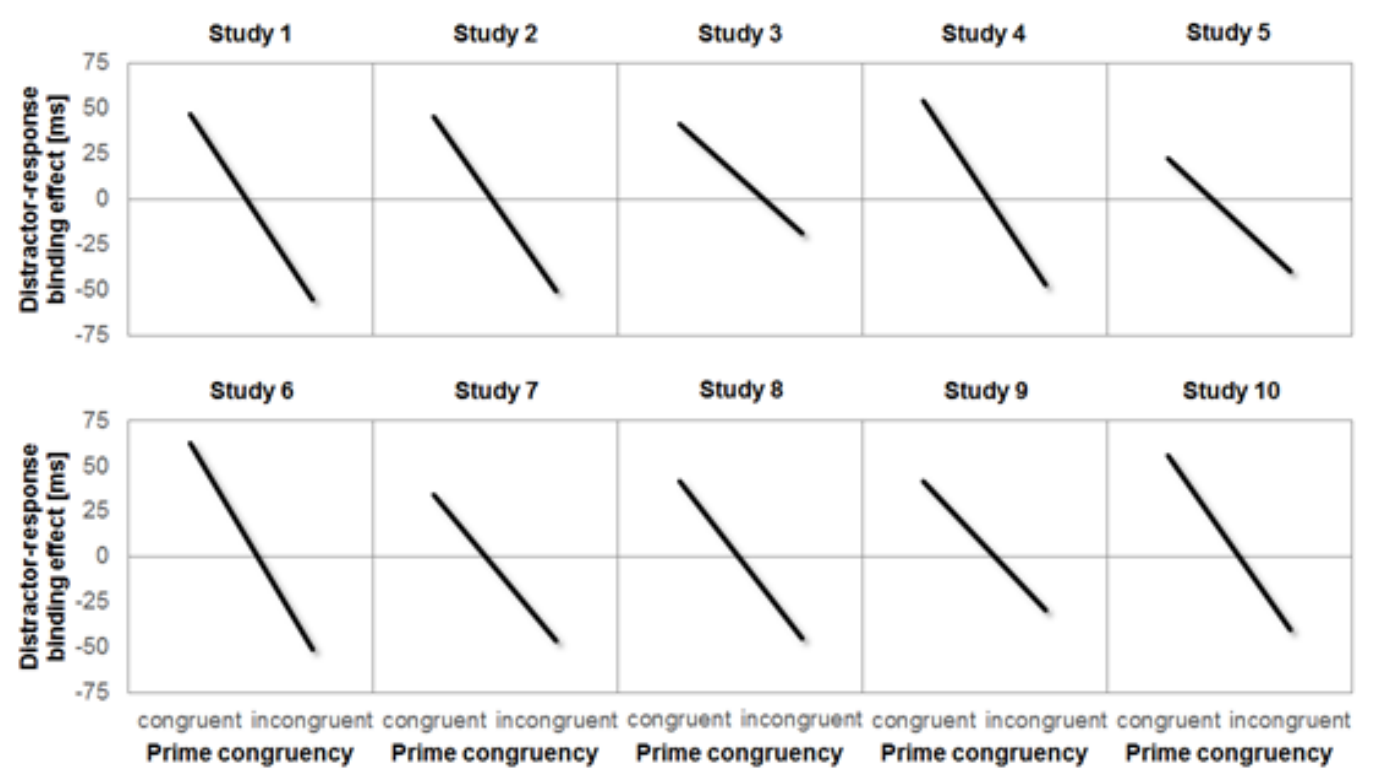

FIGURE 2.

Distractor-response binding effects computed separately for congruent and incongruent prime trials for the first 10 studies of the simulated data set (the remaining studies show the same stable pattern). Results conform to the derivation in Figure 1, with the difference between the two distractor-response binding effects being four times as large as the corresponding congruency effect.

pared to a condition with neutral distractors (which allow for an orthogonal manipulation just as well) to gauge the impact of incongruency (for results suggesting no impact of incongruent relative to neutral distractors, see Giesen, Frings \& Rothermund, 2012). Alternatively, it is possible to vary the degree of incongruency (i.e., distractors that are arbitrarily mapped to targets' responses in the context of the experiment vs. distractors that are strongly associated with targets' responses on a long-term basis) to assess how much a strong long-term stimulus-response-association can hinder binding of new distractor-response pairings in action control (see Moeller \& Frings, 2017b).

\section{CONCLUSIONS}

Following the above argument, we suggest that computing distractorresponse binding separately for congruent and incongruent prime (or probe) trials does not inform about a possible moderating role of targetdistractor congruency for event-file binding. This is not to say that such a theoretically plausible influence does not exist, but previous results reported in this direction should be treated with caution. A possible solution for this issue is to draw on studies that focus exclusively on incongruent or neutral distractors to assess the effect of incongruence on binding effects. 


\section{ACKNOWLEDGEMENTS}

This publication was funded by the German Research Foundation (DFG) and the University of Wuerzburg in the funding programme Open Access Publishing

\section{FOOTNOTES}

${ }^{1}$ It is possible to distinguish three relevant kinds of (in)congruency or (in)compatibility if distractors and targets are drawn from the same item pool. First, there can be a match or mismatch between the response that is associated with the distractor and the correct targetresponse. Second, there can be a match or mismatch between the distractor stimulus and the target stimulus due to similar or dissimilar perceptual features. Third, if there is dimensional overlap between distractor features and response features, the distractor can be (in)compatible with the target-response (e.g., due to spatial features inherent in the distractor stimulus). Since these aspects often vary together in the typical design, we will more generally refer to (in)congruent primes/ probes in the following.

${ }^{2} \mathrm{~A}$ classical way to circumvent this issue for incongruent trials is to map multiple stimuli to each response (e.g., Bertelson, 1965; Eriksen \& Eriksen, 1975). This solution cannot be applied to congruent trials for logical reasons, however: If a congruent prime is repeated and the response is repeated as well, then the probe trial will always be congruent.

${ }^{3}$ Note that comparing distractor-response binding effects for congruent and incongruent probes entails a slightly different issue (especially for the case of tasks with two response options). Congruent probes are always preceded by congruent primes if both response- and distractor-sequences repeat (or alternate), and by incongruent primes if only one of them alternates. By contrast, incongruent probes are preceded by incongruent primes if both response and distractor repeat (or alternate) and by congruent primes if only one of them alternates. Hence, in this case, distractor-response binding effects, as measured in congruent and incongruent probes, are differently influenced by congruency sequence effects (Gratton, Coles, \& Donchin, 1992; Hommel, Proctor, \& Vu, 2004; Moeller \& Frings, 2014b).

${ }^{4}$ The outcome of the simulation necessarily mirrors the formal deduction displayed in Figure 1. We still found it useful and instructive to validate our considerations via simulated results.

\section{REFERENCES}

Bertelson, P. (1965). Serial choice reaction-time as a function of response versus signal-and-response repetition. Nature, 206, 217-218. doi: 10.1038/206217a0

Bogon, J., Thomaschke, R., \& Dreisbach, G. (2017). Binding time: Evidence for integration of temporal stimulus features. Attention, Perception, \& Psychophysics, 79, 1290-1296. doi:

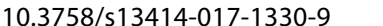

Colzato, L. S., Raffone, A., \& Hommel, B. (2006). What do we learn from binding features? Evidence for multilevel feature integra- tion. Journal of Experimental Psychology: Human Perception and Performance, 32, 705-716. Wلس سلس

Colzato, L. S., Van Wouwe, N. C., \& Hommel, B. (2007). Feature binding and affect: Emotional modulation of visuo-motor integration. Neuropsychologia, 45, 440-446. doi: 10.1016/j. neuropsychologia.2006.06.032

Davelaar, E. J. (2013). When the ignored gets bound: Sequential effects in the flanker task. Frontiers in Psychology, 3, 552. doi: 10.3389/fpsyg.2012.00552 wلس الس

Dutzi, I., \& Hommel, B. (2009). The microgenesis of action-effect binding. Psychological Research, 73, 425-435. doi: 10.1007/ s00426-008-0161-7 سلس ل1

Eriksen, B. A., \& Eriksen, C. W. (1974). Effects of noise letters upon the identification of a target letter in a nonsearch task. Perception \& Psychophysics, 16, 143-149. doi: 10.3758/ BF03203267 سلس

Frings, C. (2011). On the decay of distractor-response episodes. Experimental Psychology, 58, 125-131. doi: 10.1027/1618-3169/ a 000077 Wل

Frings, C., Koch, I., Rothermund, K., Dignath, D., Giesen, C., Hommel, B., . . \& \& Philipp, A. (in press). Merkmalsintegration und Abruf als wichtige Prozesse der Handlungssteuerung eine Paradigmen-übergreifende Perspektive [Feature integration and retrieval as core processes in action control - a crossparadigm perspective]. Psychologische Rundschau. Wلس Wل

Frings, C., \& Rothermund, K. (2011). To be or not to be...included in an event file: Integration and retrieval of distractors in stimulus-response episodes is influenced by perceptual grouping. Journal of Experimental Psychology: Learning, Memory, \& Cognition, 37, 1209-1227. doi: 10.1037/a0023915 الس الس الس

Frings, C., Rothermund, K., \& Wentura, D. (2007). Distractor repetitions retrieve previous responses to targets. The Quarterly Journal of Experimental Psychology, 60, 1367-1377. doi: 10.1080/17470210600955645 سلس

Giesen, C., Frings, C., \& Rothermund, K. (2012). Differences in the strength of distractor inhibition do not affect distractor-response bindings. Memory \& Cognition, 40, 373-387. doi: 10.3758/s13421-011-0157-1 14

Giesen, C., \& Rothermund, K. (2011). Affective matching moderates S-R binding. Cognition and Emotion, 25, 342-350. doi: 10.1080/02699931.2010.482765 سلس

Gratton, G., Coles, M. G., \& Donchin, E. (1992). Optimizing the use of information: strategic control of activation of responses.

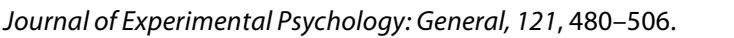
Herwig, A., \&Waszak, F. (2012). Action-effect bindings and ideomotor learning in intention-and stimulus-based actions. Frontiers in

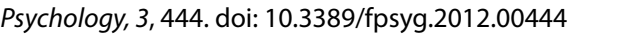

Hommel, B. (1998). Event files: Evidence for automatic integration of stimulus-response episodes. Visual Cognition, 5, 183-216. doi: 10.1080/713756773 سلس

Hommel, B. (2004). Event files: Feature binding in and across perception and action. Trends in Cognitive Sciences, 8, 494-500. 
doi: 10.1016/j.tics.2004.08.007 |سلس

Hommel, B. (2005). How much attention does an event file need? Journal of Experimental Psychology: Human Perception and Performance, 31,

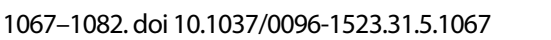

Hommel, B., Memelink, J., Zmigrod, S., \& Colzato, L.S. (2014). Attentional control of the creation and retrieval of stimulusresponse bindings. Psychological Research, 78, 520-538. doi: 10.1007/s00426-013-0503-y الس السلس

Hommel, B., Proctor, R.W., \&Vu, K.-P.L. (2004). A feature-integration account of sequential effects in the Simon task. Psychological

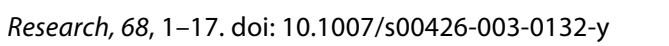

Huffman, G., Hilchey, M.D., \& Pratt, J. (2018). Feature integration in basic detection and localization tasks: Insights from the attentional orienting literature. Attention, Perception, \& Psychophysics, 80, 1333-1341. doi: 10.3758/s13414-018-1535-6 اله السلسلس

Janczyk, M., Heinemann, A., \& Pfister, R. (2012). Instant attraction: Immediate action-effect bindings occur for both, stimulusand goal-driven actions. Frontiers in Psychology, 3, 446. doi: 10.3389/fpsyg.2012.00446 السلسلس

Moeller, B., \& Frings, C. (2014a). Attention meets binding: Only attended distractors are used for the retrieval of event files. Attention, Perception, \& Psychophysics, 76, 959-978. doi: 10.3758/s13414-014-0648-9 سلس

Moeller, B., \& Frings, C. (2014b). Long term response-stimulus associations prevent distractor-response bindings. Advances in

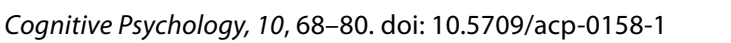

Moeller, B. \& Frings, C. (2017a). Dissociation of binding and learning processes. Attention, Perception, \& Psychophysics, 79,

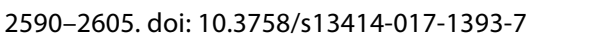

Moeller, B., \& Frings, C. (2017b). Overlearned responses hinder S-R binding. Journal of Experimental Psychology: Human Perception and Performance, 43, 1-5. Wس.

Moeller, B., Frings, C., \& Pfister, R. (2016). The structure of distractor-response bindings: Conditions for configural and elemental integration. Journal of Experimental Psychology: Human Perception and Performance, 42, 464-479. doi: 10.1037/ xhp0000158 الس الس الس

Moeller, B., Pfister, R., Kunde, W., \& Frings, C. (2016). A common mechanism behind distractor-response and response-effect binding? Attention, Perception, \& Psychophysics, 78, 1074-1086. doi: 10.3758/s13414-016-1063-1 الس

Moeller, B., Rothermund, K., \& Frings, C. (2012). Integrating the irrelevant sound - Grouping modulates the integration of irrelevant auditory stimuli into event files. Experimental Psychology,

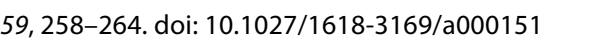

Mordkoff, J. T. (2012). Three reasons to avoid having half of the trials be congruent in a four-alternative forced-choice experiment on sequential modulation. Psychonomic Bulletin \& Review, 19, 750-757. doi: 10.3758/s13423-012-0257-3 السلسلسلس

Rothermund, K., Wentura, D., \& De Houwer, J. (2005). Retrieval of incidental stimulus-response associations as a source of negative priming. Journal of Experimental Psychology: Learning, Memory, and Cognition, 31, 482-495. doi: 10.1037/02787393.31.3.482 سلس

Schwarz, K. A., Burger, S., Dignath, D., Kunde, W., \& Pfister, R. (2018). Action-effect binding and agency. Consciousness and Cognition,

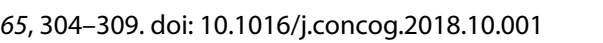

Wiswede, D., Rothermund, K., \& Frings, C. (2013). Not all errors are created equally: Specific ERN responses for errors originating from distractor-based response retrieval. European Journal of Neuroscience, 38, 3496-3506. doi: 10.1111/ejn.12340 الس الس الس 